\title{
Design and Construction of a cylindrical GEM detector as the Inner Tracker device of the KLOE-2 experiment
}

\author{
G. Morello* \\ LNF-INFN, Frascati, Italy \\ E-mail: morello@lnf.infn.it
}
A. Balla, G. Bencivenni, S. Cerioni, P. Ciambrone, E. De Lucia, D. Domenici, J. Dong, G. Felici, M. Gatta, M. Jacewicz, S. Lauciani, V. Patera, M. Pistilli, L. Quintieri, E. Tshadadze $L N F-I N F N$
G. De Robertis, G. Fanizzi, N. Lacalamita, R. Liuzzi, F. Loddo, M. Mongelli, A. Ranieri, V. Valentino
INFN Bari, Bari, Italy

\section{Schioppa}
INFN Cosenza, Gruppo collegato di Frascati, Italy

\author{
A. Di Domenico, M. Capodiferro, A. Pelosi \\ INFN Roma, Roma, Italy
}

\begin{abstract}
An Inner Tracker is under construction for the KLOE-2 experiment at the DAФNE collider. The tracker, based on GEM technology, will improve the vertex resolution near the interaction point, thanks to the good position resolution and the low material budget. Here follows a description of the detector and some measurements performed.
\end{abstract}

8th International Conference on Nuclear Physics at Storage Rings-Stori11,

October 9-14, 2011

Laboratori Nazionali di Frascati dell'INFN, Italy

\footnotetext{
* Speaker.
} 


\section{The KLOE-2 experiment}

The KLOE experiment [1] has collected an integrated luminosity of $\int \mathscr{L} d t=2.5 \mathrm{fb}^{-1}$ at the Frascati $\phi$-factory DA $\Phi \mathrm{NE}$, an $e^{+} e^{-}$collider operating at the center-of-mass energy of $1020 \mathrm{MeV}$. The experiment achieved several important results in kaon and hadronic physics. The collider has been upgraded with the aim of increasing the luminosity of about a factor 3 up to $5 \cdot 10^{32} \mathrm{~cm}^{2} \mathrm{~s}^{-1}$. The KLOE collaboration has therefore planned a new data taking period and an upgraded detector. The physics program [2] will focus on neutral kaon interferometry, study of the decays of $K_{S}, \eta$ and $\eta^{\prime}$ and searches for exotics. In the present KLOE apparatus, the tracking is provided by a huge and transparent Drift Chamber [3], filled with $\mathrm{He}: \mathrm{iC}_{4} \mathrm{H}_{10}$ 90:10. The chamber has a full stereo geometry, with a spatial resolution of $\sigma_{r \varphi} \simeq 150 \mu \mathrm{m}, \sigma_{z} \simeq 2 \mathrm{~mm}$ and a vertex resolution of $\sim 6 \mathrm{~mm}$. The resolution on transverse momenta is $\sigma_{p_{\perp}} / p_{\perp} \sim 0.4 \%$. The chamber is $4 \mathrm{~m}$ long, with a $2 \mathrm{~m}$ outer radius and $25 \mathrm{~cm}$ inner radius. It is surronded by an electromagnetic calorimeter [4], covering $98 \%$ of the solid angle. The calorimeter is composed by lead-scintillating fiber and it provides an energy resolution of $\sigma_{E} / E=5.7 \% / \sqrt{E(\mathrm{GeV})}$ and a time resolution of $\sigma_{t}=54 \mathrm{ps} / \sqrt{\mathrm{E}(\mathrm{GeV})} \oplus 100 \mathrm{ps}$. The whole apparatus is embedded in a superconductive magnet which provides a $0.52 \mathrm{~T}$ axial magnetic field. In order to improve the resolution on the vertices occuring within few $\mathrm{cm}$ from the IP, a new tracking subdetector will be installed in the free space between the Drift Chamber inner wall and the beam pipe, in this way reducing the present track extrapolation length. The Inner Tracker (IT) contribution to the overall material budget has to be carefully taken into account in order to minimize multiple scattering contribution to the track momentum resolution and the probability of the photon conversions before the DC volume. The requirements for the Inner Tracker can be summarized as:

- $\sigma_{r \varphi} \sim 200 \mu \mathrm{m}$ and $\sigma_{z} \sim 500 \mu \mathrm{m}$ spatial resolution

- $5 \mathrm{kHz} / \mathrm{cm}^{2}$ rate capability

$-<2 \% X_{0}$ material budget

The adopted solution is the Cylindrical-GEM (CGEM), a triple-GEM detector composed by concentric cylindrical electrodes: cathode, 3 GEM foils and anode, acting also as the readout circuit.

\section{The Inner Tracker of KLOE-2}

The IT of KLOE-2 will be composed by four layers of cylindrical triple-GEM $700 \mathrm{~mm}$ long, each of them equipped with a two-dimensional readout. The radius of the layers will range between $13 \mathrm{~cm}$ and $23 \mathrm{~cm}$, being limited by the beam pipe and the DC inner wall. The minimum value is chosen in order to preserve the quantum interference region $K_{L}-K_{S}$ : expressing the interference term as a function of the $K_{S}$ lifetime units ( $\tau_{S} \simeq 0.6 \mathrm{~cm}$ at a $\phi$-factory), it can be shown [5] that the smallest radius for the IT should be larger than $\simeq 20 \tau_{S} \simeq 12 \mathrm{~cm}$. Including the carbon fibers support, the detector has a material budget of $\sim 1.5 \% X_{0}$. According to simulations, the Inner Tracker will improve of a factor 3 the spatial resolution on the $K_{s} \rightarrow \pi \pi$ vertex (presently $\sim 6 \mathrm{~mm}$ ). 

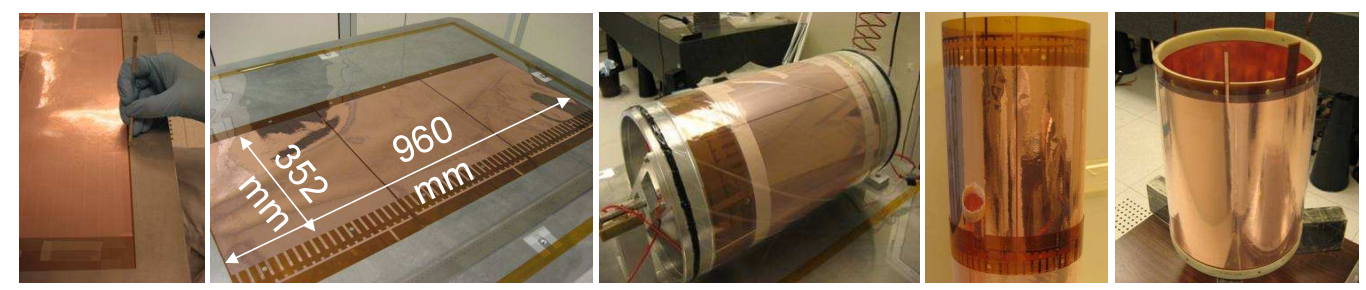

Figure 1: Steps of the construction of the CGEM prototype

\section{The cylindrical GEM prototype}

In order to validate the idea of the cylindrical GEM, a full scale radial prototype $(300 \mathrm{~mm}$ diameter and $352 \mathrm{~mm}$ active length) was built at LNF. The reduced active length of the detector was due to the unavailability, at that time, of large GEM foils. The detector is composed by five coaxial cylindrical electrodes, all made of kapton: the cathode, the three GEM stages and the anode readout. Each cylindrical electrode is realized gluing three identical foils, applying epoxy on a $3 \mathrm{~mm}$ wide region. The large foil is then wrapped on an aluminium mould coated with a very precisely machined $400 \mu \mathrm{m}$ thick Teflon film. In order to keep the electrode on the mould and during the curing cycle of the epoxy, a vacuum bag system is then used to obtain the cylindrical shape. Two fiberglass annular rings are glued on the edges of the electrodes working as spacers and mechanical support for the detector. Then the cylindrical foil can be extracted from the mould thanks to the low friction Teflon surface. Once realized, the prototype was tested at CERN-PS T9 area [7], [8] with a $10 \mathrm{GeV}$ pions beam. The detector, filled with $\mathrm{Ar}: \mathrm{CO}_{2}$ 70:30 gas mixture at 1 bar pressure, was operating at a gain of $2 \cdot 10^{4}$. The readout was formed by axial strips, $650 \mu \mathrm{m}$ pitch, and it was equipped with a FEE dedicated chip: GASTONE (see sec. 4). The detection efficiency was measured as a function of the impact parameter of the tracks (fig. 2 (left)). In fig. 2 (right) the residuals of the cluster position with respect to the tracks reconstructed by two external drift tube (DT) stations are shown. The CGEM spatial resolution is obtained by the $\sigma_{D T}$ and the $\sigma_{\text {res }}$ using $\sigma_{G E M}=\sqrt{\sigma_{r e s}^{2}-\sigma_{D T}^{2}}$. A value of $\sim 200 \mu \mathrm{m}$ arises, in agreement with what expected from a $650 \mu \mathrm{m}$ pitch readout with digital FEE.

\section{The final $X-V$ readout and the FEE}

The cylindrical shape of the detector requires a dedicated layout, since all the strips must be read out on the edges of the detector so that the X-Y orthogonal strips are not compatible. The final IT readout is performed with an X-V pattern of strips (fig. 3) engraved on a polymide foil substrate. The $\mathrm{X}$ strips with $650 \mu \mathrm{m}$ pitch will provide the $r-\phi$ coordinate while the $\mathrm{V}$ strips are realized with pads, connected through internal vias to supply the second coordinate. The Front-End Electronics of the IT is based on the GASTONE ASIC [9], a 64-channels chip featured by a low input equivalent noise and a low power consuption ( $\sim 200 \mathrm{~W}$ for 30000 channels), composed by four different stages: a charge preamplifier with $20 \mathrm{mV} / \mathrm{fC}$ sensitivity, a shaper, a leading-edge discriminator with a programmable threshold and a monostable stretcher of the digital signal, to synchronize with the KLOE Level1 trigger. 

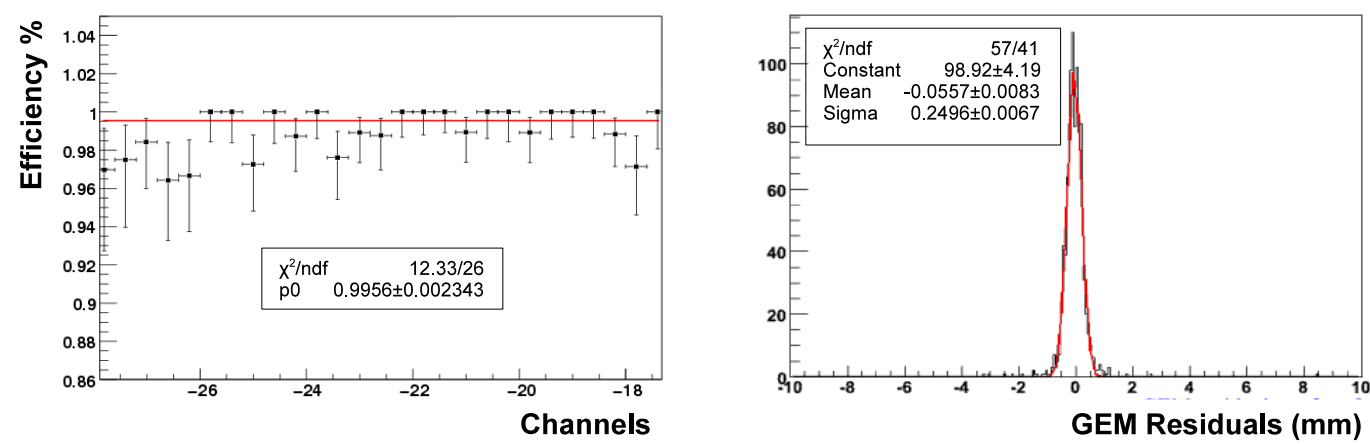

Figure 2: Detection efficiency (left) and residuals distribution (right) measured by the CGEM prototype at CERN-T9 are test beam
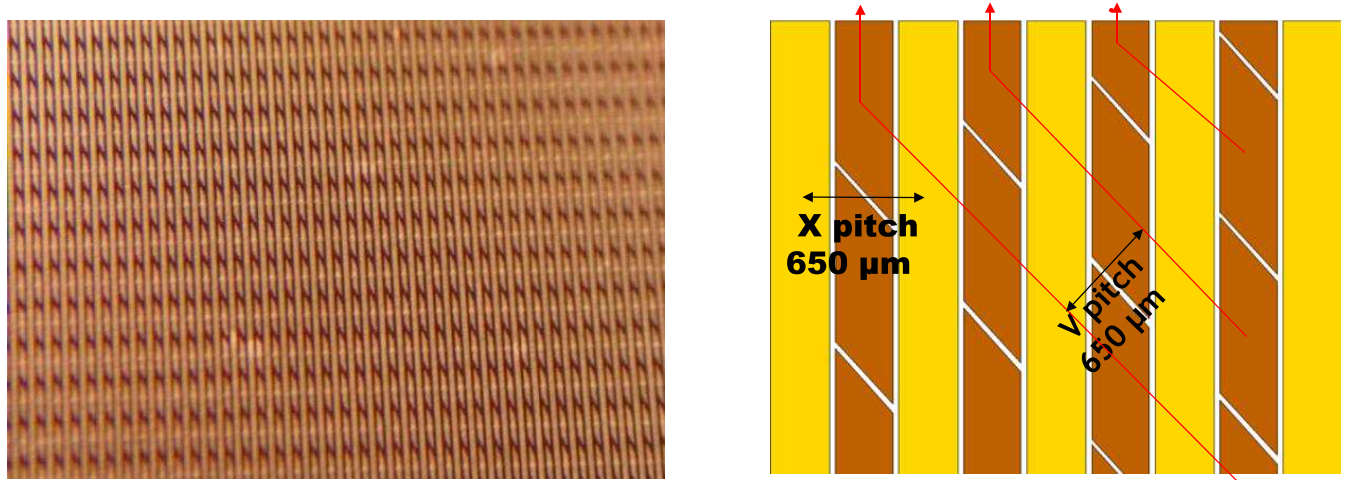

Figure 3: Detail of the orientation of the strips and pads in the final IT readout.

\section{Operation in magnetic field}

In order to simulate the working conditions in KLOE, five small $\left(10 \times 10 \mathrm{~cm}^{2}\right)$ planar tripleGEM chambers were tested in magnetic field [11] at H4 beam line (CERN-SPS), a semi-permanent RD51 facility [10]. Four chambers were equipped with orthogonal strips (X-Y readout) and used as external trackers, while for the fifth chamber the X-V readout was adopted (angle between the strips of the two directions $\sim 40^{\circ}$, fig. 3). The magnetic field was provided by GOLIATH dipole and it could be set up to $1.5 \mathrm{~T}$ in a $3 \times 3 \times 1 \mathrm{~m}^{3}$ volume. All the chambers were filled with $\mathrm{Ar}: \mathrm{CO}_{2}$ $70: 30$ at 1 bar, operating at a gain of $2 \cdot 10^{4}$. They were placed in such a way that the magnetic field was orthogonal to the electric fields inside the chambers (fig. 4), producing two effects in the GEM: a systematic displacement of the electrons with respect to the tracks position and a larger spread of the electronic cloud with respect to the no-field case. The electron shift was measured reversing the $\mathrm{X}-\mathrm{V}$ chamber with respect to the others: the shift is mirrored and the distance bewteen the spare hit and the reconstructed track is twice the displacement expected in a chamber. This quantity 


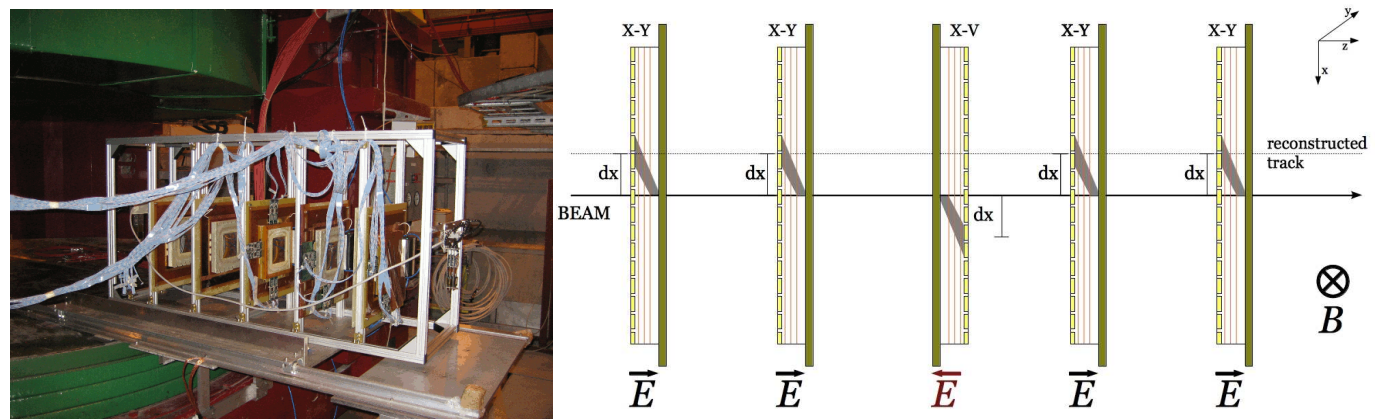

Figure 4: Layout of the chambers in the test beam at $\mathrm{H} 4$ area.

was measured as a function of the magnetic field (fig. 5), for detector electric fields set up to $1.5 / 2.5 / 2.5 / 4 \mathrm{kV} / \mathrm{cm}$. The detection efficiency as a function of the magnetic field and of the working point of the GEM was measured and is shown in fig. 5. The increase of the magnetic field, inducing a spread of the charge on the readout, results in an efficiency drop that can be recovered with higher gain. The informations on the spatial resolution along two directions ( $\mathrm{X}$ and $\mathrm{Y}$ ) are also shown in fig. 5.

\section{The Large Area GEM}

The outermost layer of the IT requires a $1440 \times 700 \mathrm{~mm}^{2} \mathrm{GEM}$ foil which is obtained splicing three $480 \times 700 \mathrm{~mm}^{2}$ foils. The request for large GEM foils led to a change of the manufacturing procedure by CERN TS-DEM-PMT laboratory, switching from the double-mask (requiring a very fine alignement of the two masks) to single-mask etching [12]. The holes of the new GEMs have quasi-cylindrical shape and for this reason a new characterization was necessary. A triple-GEM was realized with the new large foils and tested with a ${ }^{137} \mathrm{Cs}$ source (660 keV photons). The measured current was normalized to the one obtained by a $10 \times 10 \mathrm{~cm}^{2}$ double-mask manufactured triple-GEM. The measurement (fig. 6) shows that the gain for the new hole shape is $\sim 20 \%$ lower than in the double-mask case at a fixed HV. The large area triple-GEM was then tested at CERN-PS T9 area, using the same external tracking of the previous test-beam (see sec. 5). The chamber was equipped with the final X-V readout. The test beam allowed to validate the final DAQ and FEE chain, composed by the GASTONE64 chip, interface boards, General Intermediate Boards and the Software Interface. The detection efficiency as a function of the voltage applied to the GEM foils is shown in fig. 7 (right).

\section{Construction}

The construction philosophy and the toolings of the CGEM layers composing the IT are the same used for the prototype. The main construction steps can be summarized as follows: 

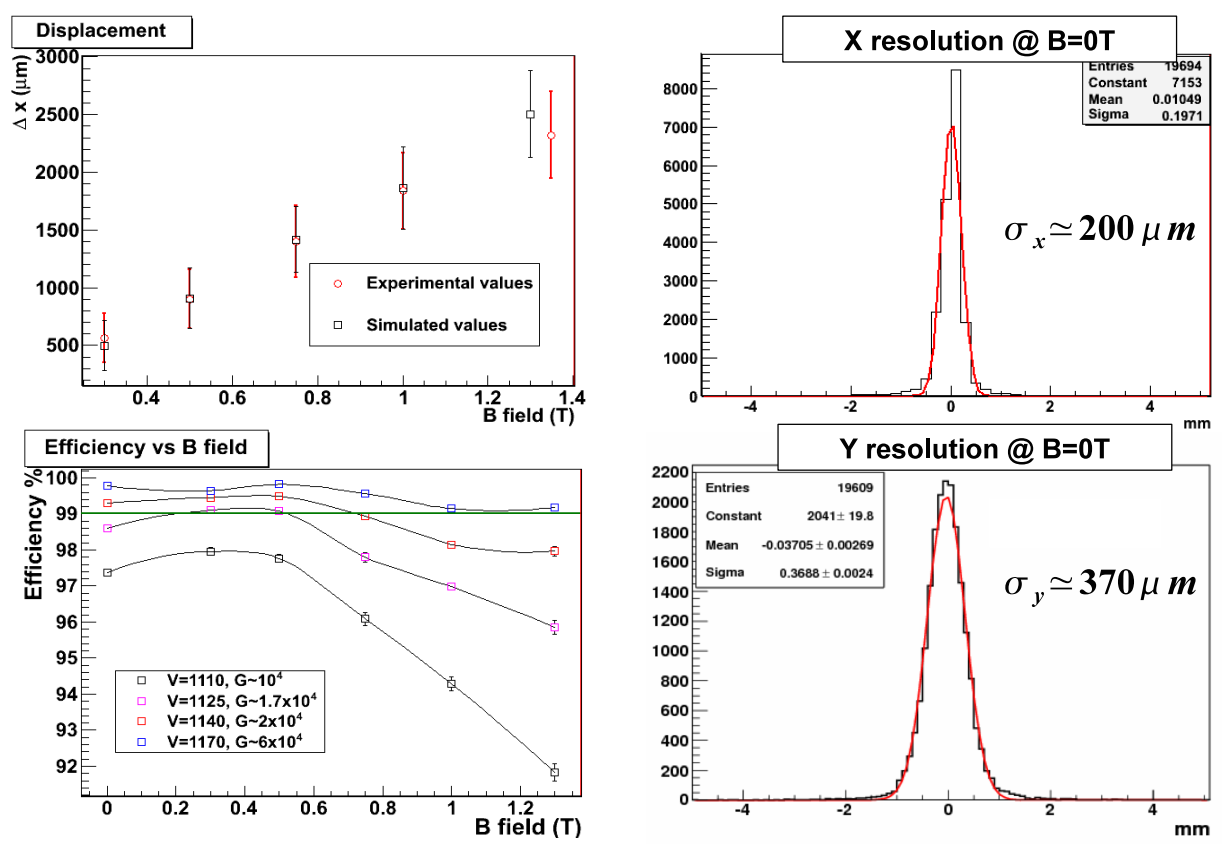

Figure 5: Electron displacement as a function of the magnetic field (top left). Spatial resolution along the $X$ direction at $0 \mathrm{~T}$ (top right). Detection efficiency as a function of the magnetic field for different gain of the $\mathrm{X}-\mathrm{V}$ chamber (bottom left). Spatial resolution along the direction orthogonal to $\mathrm{X}$ at $0 \mathrm{~T}$ (bottom right).
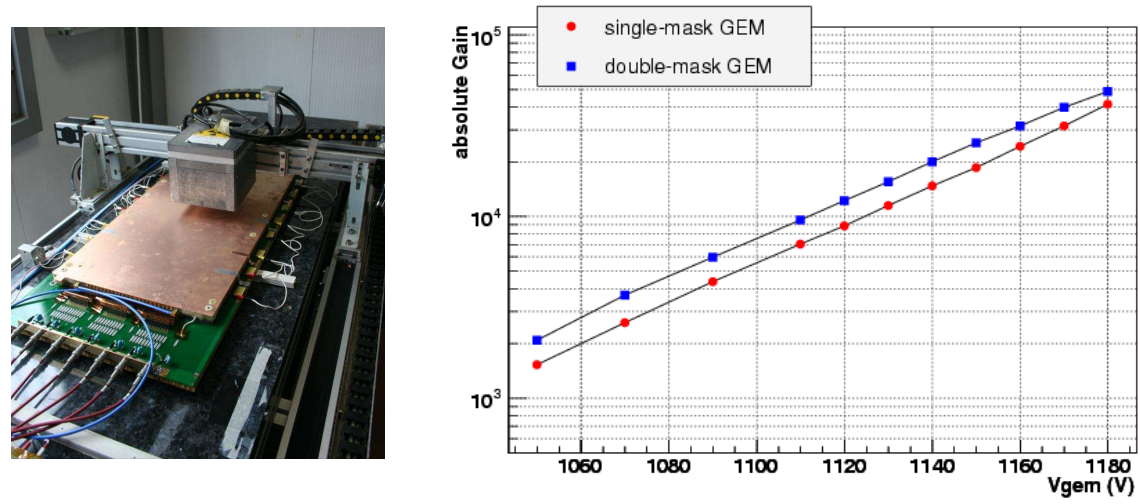

Figure 6: Setup for the measurement with the ${ }^{132} \mathrm{Cs}$ on the large planar triple-GEM and comparison of the gain measured with a double-mask and a single-mask manufactured GEM as a function of the applied voltage. 

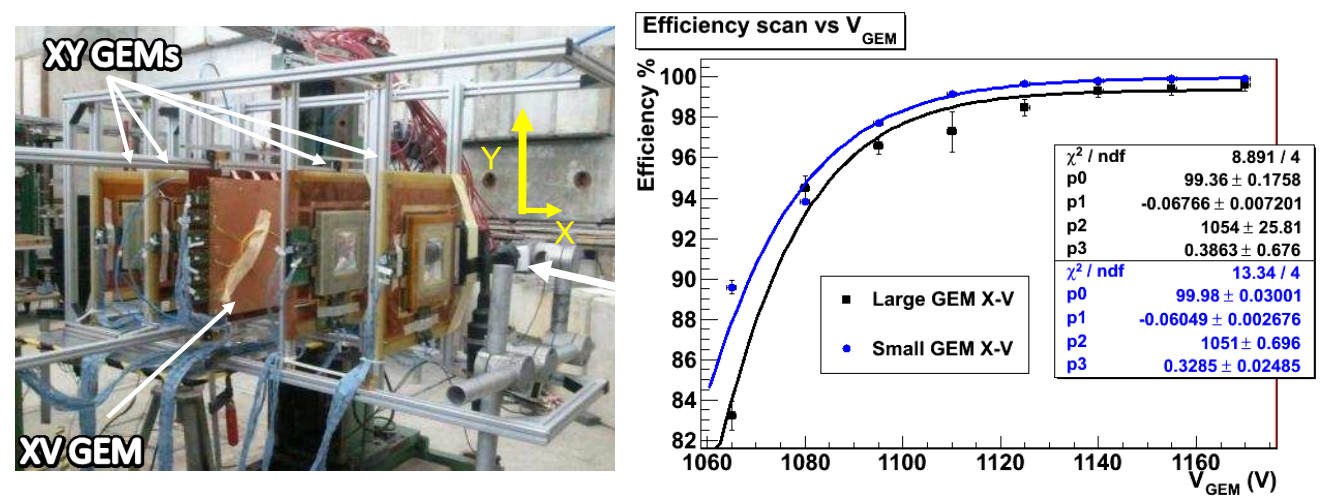

Figure 7: Setup of the Large Area GEM test beam (left) and detection efficiency as a function of the sum of the voltage applied to the GEM foils of a triple-GEM (right). The blue points are obtained by the double-mask manufactured GEMs while the black ones refer to the GEM realized with the new single-mask technique. The fit is obtained by Fermi-Dirac functions.

1. three GEM, as well as the anode (fig. 8 (left)) and cathode, foils are preliminary glued together in order to obtain a single large foil needed to realize a cylindrical electrode. For this operation we exploit a precise Alcoa plane and the vacuum bag technique.

2. the large foil is then rolled on a very precise aluminum cylindrical mould (fig. 8 (center)) covered with a $0.4 \mathrm{~mm}$ machined Teflon film for easy and safe extraction of the cylindrical electrode. The mould is then enveloped with the vacuum bag, and vacuum is applied for the glue curing time (about 12 hours).

3. The final assembling of a CGEM layer is performed by means of the Vertical Insertion System (VIS), a tool that allows a smooth and safe insertion of the cylindrical electrodes one after the others (fig. 8 (right)). The system is designed to permit a very precise alignement ( $0.1 \mathrm{~mm}$ on $1 \mathrm{~m}$ global length) of the cylindrical electrodes along their vertical axis. The bottom electrodes is fixed on its mould, while the top one is slowly moved downwards by a computer controlled step-motor, coupled with a reduction gear system. The operation is performed with the help of a web-camera, thus allowing the monitoring of the radial distance between the electrodes $(2-3 \mathrm{~mm}$ typically). The up-down rotation of the assembly tool allows an easy sealing of the detector on both sides.

\section{Conclusions}

The construction, operation and extensive test of an almost full-size CGEM prototype demonstrated the feasibility of such a novel light and dead-zone-free tracker. The final readout configuration was validated with the test of small planar prototypes operating in magnetic field. The results match the final IT requirements. A large planar GEM prototype was realized with the new singlemask technique: a characterization with $\mathrm{X}$-rays revealed that the gain is $\sim 20 \%$ lower than using 


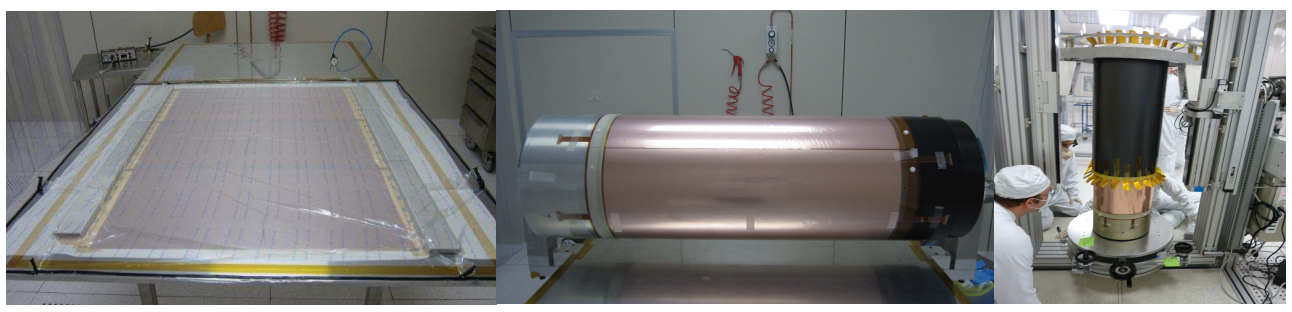

Figure 8: Planar gluing of the three anode foils (left). A Cylindrical GEM (center). Insertion of a CGEM into the anode (right).

the double-mask technique. The large GEM was then tested with the final DAQ and FEE chain that includes the GASTONE 64-channels release. The construction has started and it will be completed at the end of summer 2012.

\section{References}

[1] F.Bossi et al. and KLOE collaboration, Precision Kaon and Hadron Physics with KLOE, Rivista del Nuovo Cimento, Vol. 31, N. 10 (2008)

[2] G. Amelino Camelia et al., Physics with the KLOE-2 experiment at the upgraded DAФNE, Eur. Phys. J. C 68619 (2010)

[3] M. Adinolfi et al., The tracking detector of the KLOE experiment, NIMA 488 (2002) 51

[4] M. Adinolfi et al., The KLOE electromagnetic calorimeter, NIMA 482 (2002) 364

[5] KLOE-2 collaboration, TDR of Inner Tracker for KLOE-2 experiment, arXiv:1002.2572 and LNF-10/3(P) INFN-LNF, Frascati, 2010

[6] G. Bencivenni et al., An ultra-light cylindrical GEM based vertex detector NIMA 572 (2007) 168

[7] G. Bencivenni et al., Performance of the cylindrical GEM prototype for the KLOE-2 Inner Tracker, NSS Conf. Rec. Vol. I, 2268 (2009)

[8] G. Bencivenni et al., The full scale prototype of the cylindrical-GEM detector as Inner Tracker in KLOE-2, NSS Conf. Rec., 2007 IEEE Vol. 6, pp. 4666-4670

[9] A. Balla et al., GASTONE: a new ASIC for the cylindrical GEM Inner Tracker of KLOE experiment at $D A \Phi N E$, NIMA 604 (2009) 23

[10] M. Alfonsi et al., $R$ D Proposal. Development of Micro-Pattern Gas Detector Technologies, CERN-LHCC-2008-011/LHCC-P-001 (2008)

[11] E. De Lucia at al., Status of the cylindrical-GEM project for the KLOE-2 Inner Tracker, NIMA 628 (2011) 194

[12] M. Alfonsi et al., Activity of CERN and LNF groups on large area GEM detectors, NIM A 617 (2010) 151 